\title{
Non-random mating for selection with restricted rates of inbreeding and overlapping generations
}

\author{
Anna K. Sonesson*, Theo H.E. Meuwissen \\ Institute for Animal Science and Health (ID-Lelystad), P.O. Box 65, \\ 8200 AB Lelystad, The Netherlands
}

(Received 24 November 2000; accepted 8 August 2001)

\begin{abstract}
Minimum coancestry mating with a maximum of one offspring per mating pair (MC1) is compared with random mating schemes for populations with overlapping generations. Optimum contribution selection is used, whereby $\Delta F$ is restricted. For schemes with $\Delta F$ restricted to $0.25 \%$ per year, 256 animals born per year and heritability of 0.25 , genetic gain increased with $18 \%$ compared with random mating. The effect of $\mathrm{MC} 1$ on genetic gain decreased for larger schemes and schemes with a less stringent restriction on inbreeding. Breeding schemes hardly changed when omitting the iteration on the generation interval to find an optimum distribution of parents over age-classes, which saves computer time, but inbreeding and genetic merit fluctuated more before the schemes had reached a steady-state. When bulls were progeny tested, these progeny tested bulls were selected instead of the young bulls, which led to increased generation intervals, increased selection intensity of bulls and increased genetic gain (35\% compared to a scheme without progeny testing for random mating). The effect of $\mathrm{MC} 1$ decreased for schemes with progeny testing. MC1 mating increased genetic gain from $11-18 \%$ for overlapping and $1-4 \%$ for discrete generations, when comparing schemes with similar genetic gain and size.
\end{abstract}

mating/overlapping generations/selection/rate of inbreeding/genetic response/optimum contribution

\section{INTRODUCTION}

For breeding schemes, the selection step determines the increase in average coancestry of the population, but the mating step can improve the genetic structure of the population for the next round of selection. Caballero et al. [3] concluded that non-random mating decreased the rate of inbreeding $(\Delta F)$, but had little effect on genetic response for BLUP and phenotypic selection. A reduction of $\Delta F$ is, however, not expected when selection is made with

* Correspondence and reprints

E-mail: a.k.sonesson@id.wag-ur.nl 
a restriction on $\Delta F$, but instead the improved family structure due to nonrandom mating can be used to increase the selection differential, i.e. to increase genetic response [15]. When using optimum contribution with a restriction on $\Delta F$ and discrete generations, minimum coancestry mating with only one progeny per mating pair (MC1) increased genetic response from 5 through $23 \%$ compared with random mating. The increase in genetic response was higher when schemes were small or when the restriction on rate of inbreeding was more stringent and can be explained by the effects of $\mathrm{MC} 1$ on the relationship structure of the population. Minimum coancestry mating connects unrelated families and avoids extreme relationships. Factorial mating schemes [17] avoid extreme relationships by exchanging full-sib relationships for (maternal) half-sib relationships, which is also done in the MC1 scheme by restricting the number of progeny per mating pair to zero or one. Both these effects result in a population with more homogenous relationships among animals. Because MC1 mating makes relationships more homogenous across families, the relationship among animals with the highest $E B V \mathrm{~s}$ will be reduced. Hence, it will be easier for optimum contribution selection to select animals with the highest $E B V \mathrm{~s}$ when $\mathrm{MC1}$ mating is used instead of random mating. A third effect of non-random mating, especially minimum coancestry mating, are decreased inbreeding levels of the progeny and thus also of parents of the next generation, which leads to a larger Mendelian sampling variance. A larger Mendelian sampling variance leads to increased genetic variance and genetic gain.

The above schemes were tested for populations that had discrete generations. Most practical schemes however have overlapping generation structure. For schemes with overlapping generations, parents are selected from several ageclasses, which makes their pedigrees more heterogeneous, so that the above mentioned effects of $\mathrm{MC} 1$ mating on the relationship structure of the population are advantageous. On the contrary, benefits of $\mathrm{MC1}$ decrease with increasing population size [15] and schemes with overlapping generations can be larger than schemes with discrete generations. Hence, it is not clear how large the benefits of $\mathrm{MC} 1$ mating will be for schemes with overlapping generations.

The aim of this study was to compare genetic gain, generation interval and number of sires and dams selected for random and minimum coancestry mating with only one progeny per mating pair. Optimum contribution selection will be used for schemes with overlapping generations and with or without progeny testing of sires. For schemes with overlapping generations, the algorithm described in [12] iterates to obtain the optimum distribution of parents over age-classes. The iteration process is rather computer intensive, and we also studied the effect of omitting this iteration process and using the distribution of parents over age-classes of the previous year. Note that this does not result in a constant distribution of parents over age-classes, because the distribution 
defined by the contribution of each animal may deviate from the distribution of the last year. Finally, we compared schemes with overlapping and discrete generations to see where minimum coancestry mating performs the best.

\section{MATERIALS AND METHODS}

\subsection{Optimum contribution selection}

The optimum contribution selection method for discrete generations was presented by Meuwissen [11] and for overlapping generations by Meuwissen and Sonesson [12]. In principle, the genetic response is maximised with two restrictions. Firstly, there is a restriction on the contribution per sex, i.e. each sex has to contribute $50 \%$ of the genes to the next generation. Secondly, a constraint on the increase of the average relationship of selected parents limits the inbreeding of each selection round. For populations with overlapping generations, there are animals of different age-classes at year $t$ from which parents are selected and the different age-classes get different weights, $\mathbf{r}$, which equal their long-term contributions and which are derived from the gene-flow theory of Hill [10]. The weights indicate how much age-classes are expected to contribute in the future. The algorithm iterates on these weights such that the contribution of each individual selection candidate, $\mathbf{c}_{t}$, and the contributions of each age-class, $\mathbf{r}$, are optimised. For discrete generations, only the contribution of each individual selection candidate is optimised.

This iteration process over age-classes is rather computer intensive because it results in many calculations of optimum $\mathbf{c}_{t}$. Therefore, we investigated the consequences of omitting the iteration and simply using the distribution of parents of the last year, $\mathbf{c}_{t}{ }_{1}$, over age-classes to calculate $\mathbf{r}$. This does not result in a constant distribution of parents over age-classes, because the distribution defined by $\mathbf{c}_{t}$ may deviate from the distribution of the last year, which was used to calculate $\mathbf{r}$. For year one, $\mathbf{r}$ is $1 / q$, for all age-classes, where $q$ is the number of age-classes.

\subsection{Mating}

Two mating schemes described in Sonesson and Meuwissen [15] are used.

For random mating (RAND), sires and dams are allocated at random with a probability that is proportional to the genetic contribution that they received from the selection algorithm. Note that for the random mating scheme, the actual number of offspring deviated from the optimal contributions due to sampling of parents, which introduced some suboptimality in the schemes.

The non-random mating strategy minimises the average coancestry of sires and dams, whereby the number of progeny per mating pair is restricted to zero and one (MC1). MC1 gave the highest genetic gain of all schemes in the 
study for discrete generations [15]. For the MC1 scheme, a matrix $\mathbf{F}$ of size $\left(N_{\mathrm{s}} \times N_{\mathrm{d}}\right)$ is set up, where $N_{\mathrm{s}}\left(N_{\mathrm{d}}\right)$ is the number of selected sires (dams) and $\mathbf{F}_{i j}$ is the coefficient of coancestry of selected animals $i$ and $j$, which is also the inbreeding coefficient of their progeny. The simulated annealing algorithm [14] was applied to find the mating pairs with average minimum coancestry. This algorithm implies sampling of different mating pairs from the $\mathbf{F}$ matrix and thereafter evaluating the average coancestry of every new solution. For each sample, the total number of progeny per candidate has to equal the number given from the selection algorithm, the $\mathbf{c}_{t}$ vector, which reduces the number of possible solutions (see Fig. 2 of [15]). If the average coancestry is lower than for the previous solution, the new solution is accepted. If the average coancestry is higher, the new solution is accepted with a probability that depends on the number of samples and accepted changes made. The solution vector $\mathbf{c}_{t}$ times $2 N$, rounded to integers, equals a vector of optimal number of progeny that each candidate should obtain, where $N$ is the total number of newborn progeny per year. Normally, the truncation point for rounding up versus down is 0.5 , but if the total number of progeny did not sum to $N$, the truncation point is adjusted such that $N$ progeny result. This is not guaranteed to give the optimum integer solution, but it probably yields a reasonable approximation for these relatively large schemes. See Appendix of [15] for a more detailed explanation of the MC1 algorithm.

\subsection{Selection schemes}

The parameters of the simulated breeding schemes are given in Table I. The general structure was that of a closed nucleus breeding scheme for dairy cattle, i.e. selection is for a sex-limited trait, which is recorded during the reproductive age. The test daughters of progeny tested bulls came from unrelated dams outside the nucleus. Genotypes, $g_{i}$, for base animals were sampled from a distribution $\mathrm{N}\left(0, \sigma_{\mathrm{a}}^{2}\right)$, where $\sigma_{\mathrm{a}}^{2}$ is the base generation genetic variance (Tab. I). Later years were obtained by simulating progeny genotypes from $g_{i}=\frac{1}{2} g_{\mathrm{s}}+\frac{1}{2} g_{\mathrm{d}}+m_{i}$, where $\mathrm{s}$ and d denote sire and dam of progeny $i$ and $m_{i}$ equals the Mendelian sampling component, which is sampled from $\mathrm{N}\left(0, \frac{1}{2}(1-\bar{F}) \sigma_{\mathrm{a}}^{2}\right)$, where $\bar{F}$ is the average inbreeding coefficient of parents $\mathrm{s}$ and $\mathrm{d}$. For overlapping generations, phenotypes are simulated by adding terms for permanent, $\mathrm{pe}_{i}$, and temporary environment, $\mathrm{te}_{i}$, which are sampled from the distribution $\mathrm{N}\left(0, \sigma_{\mathrm{pe}}^{2}\right)$ and $\mathrm{N}\left(0, \sigma_{\mathrm{te}}^{2}\right)$, respectively, where $\sigma_{\mathrm{pe}}^{2}$ is the permanent environment variance and $\sigma_{\text {te }}^{2}$ is the temporary environment variance. For discrete generations, only the term for the temporary environment is added. Estimates of breeding values ( $E B V \mathrm{~s})$ are obtained by using the BLUP breeding value estimation procedure [9]. For overlapping generations, animals are selected only on their $E B V \mathrm{~s}$ during the first five years, and thereafter the 
Table I. Parameters of the closed nucleus breeding scheme.

\section{Size of selection scheme}

Number of newborn progeny per year

(males plus females)

256,512

Total number of years evaluated

Number of years before optimum selection started

20 or 30

Number of replicated simulations

5

100

Reproductive rate of males and females

unlimited

Number of reproductive age-classes considered

10

Inbreeding constraint

0.25 or $0.50 \%$ per year

\section{Genetic and permanent and temporary environmental variances of trait}

Overlapping generations

Discrete generations
$0.10,0.25$ and 0.65 or

$0.25,0.25$ and 0.50 or

$0.50,0.25$ and 0.25

$0.25,0.00$ and 0.75

\section{Parameters for schemes with overlapping generations}

Age at which females completed lactation records

Number of test daughters of bulls

for overlapping generations

Age at which progeny test became available

Involuntary culling rate of males and females

Voluntary culling rate
3,4 and 5 years ${ }^{(a)}$

0 or 100

5 years ${ }^{(a)}$

0.3

negligible

(a) When females are selected for this information, progeny are born one year later (i.e. generation interval is one year longer than the age at which the information becomes available).

optimum contribution algorithm is used for another 15 years for schemes without the progeny test and another 25 years for schemes with the progeny test.

For the comparison between discrete and overlapping schemes, we compared genetic gain for three discrete schemes with the same (restricted) $\Delta F$ and genetic gain $(\Delta G)$ per generation as the overlapping scheme. For the discrete schemes, the number of candidates per generation, i.e. a comparable size and selection differential, was found by trial and error such that $\Delta G$ per generation equalled that of the overlapping schemes. Average results from generations 5 through 9 were used, because the discrete scheme converged to equilibrium values after about five generations. Rates of inbreeding of the discrete and overlapping schemes were equalised by the inbreeding constraints per generation. The results were based on 100 replicated schemes. 


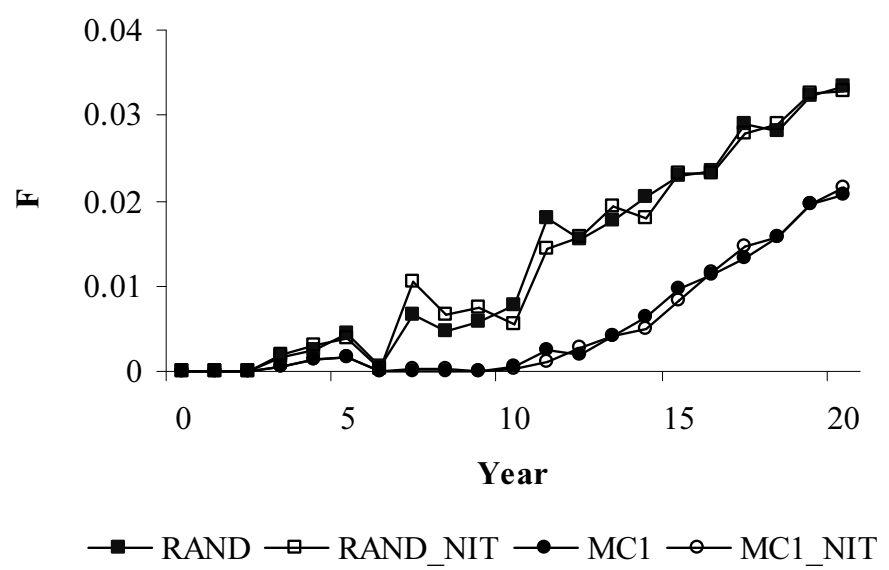

Figure 1. Inbreeding $(F)$ for schemes with $\Delta F$ restricted to $0.25 \%$, heritability of 0.25 and 256 new-born animals per year. $\mathrm{RAND}=$ random mating, $\mathrm{MC} 1=$ minimum coancestry mating with one progeny per mating pair, RAND_NIT and MC1_NIT do not iterate on generation intervals.

\section{RESULTS}

\subsection{Rate of inbreeding}

The rate of inbreeding $(\Delta F)$ did not significantly exceed the restricted level, 0.25 or $0.50 \%$ per year (Tabs. II, III, IV and V). The level of inbreeding, $F$, fluctuated more for RAND schemes than for MC1 schemes (Fig. 1). Similarly, the level of inbreeding fluctuated more for schemes without iteration on the generation interval than for schemes with iteration on the generation interval. Note that there were first five years of BLUP selection, before the scheme with a restriction on $\Delta F$ started. After the initial five years, all schemes needed about four years (one generation) before the constraint became linear. These problems in the beginning were due to different relationships of progeny of different age-classes in the first years (see also [12]).

\subsection{Genetic gain}

\subsubsection{Small scheme with low inbreeding}

Genetic gain was higher for MC1 than for RAND schemes. For schemes with a $\Delta F$ constrained to $0.25 \%$ per year, 256 newborn animals per year, heritability of 0.25 and with iteration on the generation interval, genetic gain per year, $\Delta G$, was $0.071 \sigma_{\mathrm{p}}$ units for $\mathrm{MC} 1$ and $0.060 \sigma_{\mathrm{p}}$ units for RAND mating schemes, i.e. $\Delta G$ was $18 \%$ higher for the $\mathrm{MC} 1$ schemes (Tab. II). The genetic level fluctuated during the first years after the BLUP selection, following the 
Table II. Rate of inbreeding per year $(\Delta F)$, genetic gain per year $(\Delta G)$, generation interval $(L)$ and the number of selected sires and dams $(\mathrm{Sel}$.) for schemes with $\Delta F$ restricted to $0.25 \%$ per year, 256 newborn animals per year, heritability level $\left(h^{2}\right)$ of $0.10,0.25$ or 0.50 , random or minimum coancestry mating and with or without iteration on generation interval.

\begin{tabular}{|c|c|c|c|c|}
\hline Method $^{(a)}$ & $\begin{array}{c}\Delta F(s e)^{(\mathrm{b}, \mathrm{c})} \\
(\%)\end{array}$ & $\begin{array}{c}\Delta G(s e)^{(\mathrm{b}, \mathrm{c})} \\
\left(\sigma_{\mathrm{p}} \text {-units }\right)\end{array}$ & $\begin{array}{c}L(\text { sire } / \text { dam }) \\
\quad(\text { years })\end{array}$ & Sel. sire/dam ${ }^{(\mathrm{c})}$ \\
\hline \multicolumn{5}{|c|}{$h^{2}=0.10$} \\
\hline RAND & $0.24(0.07)$ & $0.029(0.005)$ & $3.3 / 4.8$ & $33.8 / 12.5$ \\
\hline RAND_NIT & $0.23(0.06)$ & $0.028(0.005)$ & $3.2 / 4.8$ & $36.1 / 13.1$ \\
\hline $\mathrm{MC} 1$ & $0.25(0.05)$ & $0.033(0.005)$ & $3.3 / 4.7$ & $32.4 / 12.0$ \\
\hline MC1_NIT & $0.26(0.03)$ & $0.034(0.004)$ & $3.1 / 4.6$ & $34.5 / 12.7$ \\
\hline \multicolumn{5}{|c|}{$h^{2}=0.25$} \\
\hline RAND & $0.24(0.05)$ & $0.060(0.007)$ & $3.0 / 4.7$ & $35.0 / 12.0$ \\
\hline RAND_NIT & $0.23(0.05)$ & $0.062(0.007)$ & $3.1 / 4.6$ & $36.2 / 12.3$ \\
\hline MC1 & $0.24(0.04)$ & $0.071(0.007)$ & $2.9 / 4.5$ & $35.2 / 12.0$ \\
\hline MC1_NIT & $0.26(0.03)$ & $0.071(0.007)$ & $2.9 / 4.5$ & $35.3 / 11.8$ \\
\hline \multicolumn{5}{|c|}{$h^{2}=0.50$} \\
\hline RAND & $0.23(0.05)$ & $0.111(0.010)$ & $2.9 / 4.4$ & $36.2 / 11.4$ \\
\hline RAND_NIT & $0.23(0.05)$ & $0.111(0.010)$ & $2.9 / 4.4$ & $38.5 / 12.1$ \\
\hline MC1 & $0.27(0.04)$ & $0.131(0.009)$ & $2.6 / 4.3$ & $38.6 / 11.6$ \\
\hline MC1_NIT & $0.27(0.03)$ & $0.128(0.009)$ & $2.6 / 4.3$ & $37.7 / 11.6$ \\
\hline
\end{tabular}

(a) $\mathrm{RAND}=$ random mating, $\mathrm{MC1}=$ minimum coancestry mating with only one progeny per mating pair, RAND_NIT and MC1_NIT schemes do not iterate on generation interval.

(b) $s e=$ standard error.

(c) Average year 16-20.

fluctuations of the level of inbreeding (Fig. 2), i.e. genetic gain increased when the level of inbreeding increased and vice versa.

For the heritability levels of 0.10 and $0.50, \Delta G$ was 14 and $18 \%$ higher for $\mathrm{MC} 1$ schemes than for RAND schemes, respectively. Hence, the effect on $\Delta G$ of $\mathrm{MC1}$ mating is similar for different levels of heritability.

$\Delta G$ did not differ significantly for schemes with or without iteration on the generation interval.

\subsubsection{Larger scheme}

For the larger scheme (512 newborn animals per year) with $\Delta F$ restricted to $0.25 \%, \Delta G$ was $0.084 \sigma_{\mathrm{p}}$ units for the $\mathrm{MC} 1$ schemes and $0.076 \sigma_{\mathrm{p}}$ units for 


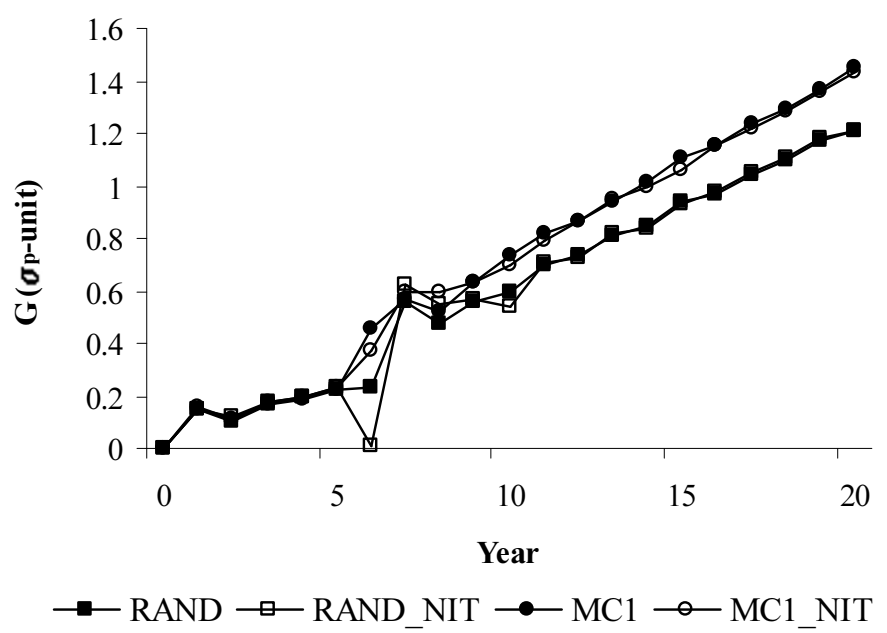

Figure 2. Genetic level $(G)$ for schemes with $\Delta F$ restricted to $0.25 \%$, heritability of 0.25 and 256 new-born animals per year. RAND = random mating, $\mathrm{MC} 1=$ minimum coancestry mating with one progeny per mating pair, RAND_NIT and MC1_NIT do not iterate on generation intervals.

Table III. Rate of inbreeding per year $(\Delta F)$, genetic gain per year $(\Delta G)$, generation interval $(L)$ and the number of selected sires and dams $(\mathrm{Sel}$.) for schemes with $\Delta F$ restricted to 0.25 or $0.50 \%$ per year, 256 or 512 newborn animals per year, heritability level of 0.25 , random or minimum coancestry mating and with or without iteration on generation interval.

\begin{tabular}{|c|c|c|c|c|}
\hline Method $^{(a)}$ & $\begin{array}{c}\Delta F(s e)^{(\mathrm{b}, \mathrm{c})} \\
(\%)\end{array}$ & $\begin{array}{c}\Delta G(s e)^{(\mathrm{b}, \mathrm{c})} \\
\left(\sigma_{\mathrm{p}} \text {-units }\right)\end{array}$ & $\begin{array}{c}L(\text { sire } / \text { dam }) \\
\quad{ }^{(\text {years })}\end{array}$ & Sel. sire/dam ${ }^{(\mathrm{c})}$ \\
\hline \multicolumn{5}{|c|}{$\Delta F=0.25 \%$ and 512 newborn animals per year } \\
\hline RAND & $0.24(0.05)$ & $0.076(0.007)$ & $2.8 / 4.6$ & $40.1 / 13.2$ \\
\hline RAND_NIT & $0.26(0.05)$ & $0.073(0.007)$ & $2.8 / 4.5$ & $39.8 / 13.5$ \\
\hline MC1 & $0.27(0.05)$ & $0.084(0.006)$ & $2.8 / 4.4$ & $42.1 / 13.9$ \\
\hline MC1_NIT & $0.27(0.04)$ & $0.086(0.006)$ & $2.8 / 4.4$ & $40.9 / 13.8$ \\
\hline \multicolumn{5}{|c|}{$\Delta F=0.50 \%$ and 256 newborn animals per year } \\
\hline RAND & $0.52(0.21)$ & $0.072(0.010)$ & $3.0 / 4.7$ & $19.4 / 6.4$ \\
\hline RAND_NIT & $0.47(0.12)$ & $0.068(0.009)$ & $2.8 / 4.6$ & $20.4 / 6.7$ \\
\hline $\mathrm{MC} 1$ & $0.52(0.16)$ & $0.082(0.010)$ & $3.1 / 4.5$ & $21.1 / 6.5$ \\
\hline MC1_NIT & $0.52(0.11)$ & $0.083(0.009)$ & 2.9/4.4 & $22.3 / 6.7$ \\
\hline
\end{tabular}

(a) RAND = random mating, $\mathrm{MC} 1=$ minimum coancestry mating with only one progeny per mating pair, RAND_NIT and MC1_NIT schemes do not iterate on generation interval. ${ }^{(b)} s e=$ standard error. ${ }^{(c)}$ Average year 16-20. 
Table IV. Rate of inbreeding per year $(\Delta F)$, genetic gain per year $(\Delta G)$, generation interval $(L)$ and the number of selected sires and dams $(\mathrm{Sel}$.) for schemes with $\Delta F$ restricted to 0.25 or $0.50 \%$ per year, 256 newborn animals per year, heritability level of 0.25 , random or minimum coancestry mating, with or without iteration on generation interval and sires are yearly progeny tested on 100 daughters outside the nucleus.

\begin{tabular}{|c|c|c|c|c|}
\hline Method $^{(a)}$ & $\begin{array}{c}\Delta F(s e)^{(\mathrm{b}, \mathrm{c})} \\
(\%)\end{array}$ & $\begin{array}{c}\Delta G(s e)^{(\mathrm{b}, \mathrm{c})} \\
\left(\sigma_{\mathrm{p}} \text {-units }\right)\end{array}$ & $\begin{array}{c}L(\text { sire/dam })^{(\mathrm{c})} \\
\quad(\text { years })\end{array}$ & Sel. sire/dam ${ }^{\text {(c) }}$ \\
\hline \multicolumn{5}{|c|}{$\Delta F=0.25 \%$} \\
\hline RAND & $0.24(0.07)$ & $0.081(0.011)$ & $5.9 / 4.3$ & $9.6 / 12.6$ \\
\hline RAND_NIT & $0.26(0.07)$ & $0.081(0.011)$ & $5.9 / 4.3$ & $9.7 / 11.6$ \\
\hline MC1 & $0.27(0.06)$ & $0.087(0.008)$ & $6.1 / 4.1$ & $7.4 / 13.9$ \\
\hline MC1_NIT & $0.25(0.06)$ & $0.088(0.008)$ & $6.1 / 4.1$ & $6.4 / 13.0$ \\
\hline \multicolumn{5}{|c|}{$\Delta F=0.50 \%$} \\
\hline RAND & $0.48(0.22)$ & $0.096(0.008)$ & $6.1 / 4.4$ & $4.6 / 6.8$ \\
\hline RAND_NIT & $0.41(0.19)$ & $0.085(0.008)$ & $6.1 / 4.3$ & $4.1 / 6.0$ \\
\hline MC1 & $0.51(0.23)$ & $0.099(0.010)$ & $6.2 / 4.1$ & $4.0 / 7.8$ \\
\hline MC1_NIT & $0.47(0.16)$ & $0.095(0.010)$ & $6.2 / 4.1$ & $4.0 / 8.0$ \\
\hline
\end{tabular}

(a) RAND = random mating, $\mathrm{MC1}=$ minimum coancestry mating with only one progeny per mating pair, RAND_NIT and MC1_NIT schemes do not iterate on generation interval.

(b) $s e=$ standard error.

(c) Average year 16-20.

the RAND schemes, i.e. $\Delta G$ was $11 \%$ higher for the MC1 schemes than for the RAND schemes. Thus, the effect on $\Delta G$ of MC1 mating was somewhat smaller for larger schemes.

\subsubsection{Less stringent restriction on inbreeding}

$\Delta G$ for schemes with a less stringent restriction on $\Delta F(0.50 \%)$ was, as expected, somewhat higher than for the scheme with a more stringent restriction on $\Delta F$ in Table II $(0.25 \%)$. For schemes with a heritability of $0.25, \Delta G$ was $0.082 \sigma_{\mathrm{p}}$ units for MC1 schemes and $0.072 \sigma_{\mathrm{p}}$ units for RAND schemes, i.e. $\Delta G$ was $14 \%$ higher for the MC1 schemes than for RAND schemes (Tab. III). Hence, the effect on $\Delta G$ of MC1 mating is somewhat smaller for schemes with a less stringent restriction on inbreeding.

Again, $\Delta G$ did not differ significantly for schemes with or without iteration on the generation interval. 
Table V. Rate of inbreeding per generation $(\Delta F /$ gen $)$, genetic gain per generation $(\Delta G / g e n)$ and number of selected sires and dams (Sel.) for schemes with discrete generations, $\Delta F$ restricted to 1 or $2 \%$ per generation, heritability level of 0.25 and random or minimum coancestry mating.

\begin{tabular}{|c|c|c|c|}
\hline Method $^{(a)}$ & $\begin{array}{c}\Delta F / \operatorname{gen}(s e)^{(\mathrm{b}, \mathrm{c})} \\
(\%)\end{array}$ & $\begin{array}{c}\Delta G / \operatorname{gen}(s e)^{(\mathrm{b}, \mathrm{c})} \\
\left(\sigma_{\mathrm{p}} \text {-units }\right)\end{array}$ & Sel. sire/dam ${ }^{(\mathrm{c})}$ \\
\hline
\end{tabular}

$\Delta F /$ gen $=1 \%, \Delta G /$ gen $=0.24$, number of animals $=324$

$\begin{array}{llll}\text { RAND } & 1.01(0.02) & 0.240(0.003) & 74.7 / 31.8 \\ \text { MC1 } & 0.96(0.01) & 0.247(0.002) & 70.9 / 28.9\end{array}$

$\Delta F /$ gen $=2 \%, \Delta G /$ gen $=0.29$, number of animals $=400$

$\begin{array}{llll}\text { RAND } & 1.99(0.04) & 0.294(0.004) & 43.2 / 18.1 \\ \text { MC1 } & 2.02(0.03) & 0.307(0.004) & 41.9 / 17.8\end{array}$

$\Delta F /$ gen $=1 \%, \Delta G /$ gen $=0.30$, number of animals $=752$

$\begin{array}{llll}\text { RAND } & 0.99(0.02) & 0.308(0.003) & 86.1 / 37.5 \\ \text { MC1 } & 0.98(0.02) & 0.312(0.003) & 79.7 / 35.8\end{array}$

(a) $\mathrm{RAND}=$ random mating, $\mathrm{MC} 1=$ minimum coancestry mating with only one progeny per mating pair.

(b) $s e=$ standard error.

(c) Average generation 5-9.

\subsubsection{Scheme with progeny test}

For schemes with progeny tests, $\Delta F$ constrained to $0.25 \%$ per year, 256 newborn animals per year, heritability of 0.25 and with iteration on the generation interval, $\Delta G$ was $0.087 \sigma_{\mathrm{p}}$ units for $\mathrm{MC} 1$ and $0.081 \sigma_{\mathrm{p}}$ units for RAND mating schemes, i.e. $\Delta G$ was $7 \%$ higher for the $\mathrm{MC} 1$ schemes. Hence, the effect on $\Delta G$ of $\mathrm{MC1}$ mating was small for schemes with progeny tests.

Again, $\Delta G$ did not differ significantly for schemes with or without iteration on the generation interval.

\subsubsection{Comparison of schemes with overlapping and discrete generations}

Three schemes were compared for discrete and overlapping schemes: one with a $\Delta F$ restricted to $0.25 \%$ per year and 256 newborn animals from Table II, one with a $\Delta F$ restricted to $0.25 \%$ per year and 512 newborn animals from Table III and one with a $\Delta F$ restricted to $0.50 \%$ per year and 256 newborn animals from Table III. The generation interval was approximately 4 years on average for all schemes and the comparison was made for the schemes with 
$h^{2}=0.25$. The number of animals needed for the discrete schemes to achieve the same genetic response per generation, i.e. same selection differential and comparable number of selection candidates, as the overlapping scheme were 324, 400 and 752, for the three schemes, respectively. For the three discrete schemes, $\Delta G$ was 3, 4 and $1 \%$ higher, respectively, for the MC1 scheme than for the RAND scheme (Tab. V). For schemes with overlapping generations, these figures were 18, 14 and $11 \%$, respectively (Tabs. II and III). Hence, the effect on $\Delta G$ of $\mathrm{MC} 1$ mating was larger for schemes with overlapping generations than for schemes with discrete generations.

\subsection{Number of selected animals and generation interval}

\subsubsection{Small scheme with low inbreeding}

For schemes with $\Delta F$ constrained to $0.25 \%$ per year, 256 newborn animals per year, heritability of 0.25 and an iteration on the generation interval, there were on average 35.2 sires and 12.0 dams selected for MC1 schemes and 35.0 sires and 12.0 dams selected for the RAND schemes (Tab. II). Dams were thus selected more intensively than sires, because of the higher accuracy of selection of cows compared to sires $[1,12]$. There was, however, no difference in the number of selected animals for the two mating schemes.

Generation intervals of the MC1 scheme for sires and dams were 2.9 and 4.5 years, respectively, which were very similar to the generation intervals of the RAND scheme of 3.0 and 4.7 years for sires and dams, respectively.

For the different levels of $h^{2}$, the same generation interval and number of selected animals were achieved with RAND and MC1 schemes. Similarly, iteration on generation intervals did not affect the breeding schemes.

\subsubsection{Larger schemes and schemes with higher inbreeding}

For the larger schemes (512 newborn animals per year) with the stringent restriction on $\Delta F(0.25 \%)$, about the same number of animals were selected (Tab. III), i.e. the selection intensity has increased for the larger schemes. For the less stringent restriction on $\Delta F(0.50 \%)$, fewer animals were selected than for the more stringent restriction $(0.25 \%)$, i.e. selection intensity also increased for this scheme with a less stringent restriction on $\Delta F$.

\subsubsection{Scheme with progeny tests}

For the scheme where sires were progeny tested on 100 daughters outside the nucleus, the progeny tested bulls were selected, which can be seen on the generation interval of the selected sires (Tab. IV). The generation interval for the sires was around 6 years for all schemes, which is the age when the information of the progeny test becomes available. The generation interval for 
the dams was around 4 years as for the schemes without progeny tests (Tabs. II and III).

For the scheme with a $\Delta F$ restricted to $0.25 \%$, the selection intensity of sires increased substantially, because the longer generation interval decreased the $\Delta F$ per year, such that there were only about 8 sires selected (Tab. III), compared with the scheme without progeny tests, where around 35 sires were selected (Tab. II).

\subsubsection{Comparison of schemes with overlapping and discrete generations}

For the scheme with discrete generations, there were somewhat fewer females selected for the $\mathrm{MC} 1$ scheme in order to get the same selection intensity as the RAND scheme. For example, for the scheme with $\Delta F$ restricted to $1 \%$ per generation and 324 newborn animals per year, there were only 70.9 males and 28.9 females selected for the MC1 schemes, whereas there were 74.7 males and 31.8 females selected for the RAND schemes.

\section{DISCUSSION}

\subsection{Comparison between RAND and MC1 matings}

The MC1 mating scheme, which minimises the coancestry of mating pairs, resulted in $18 \%$ higher $\Delta G$ than random mating schemes with a $\Delta F$ restricted to 0.25 per year, 256 newborn animals per year and $h^{2}=0.25$ (Tab. II). With larger schemes (512 newborn animals per year) or a less stringent restriction on $\Delta F$ ( $0.5 \%$ per year), the advantage of MC1 was somewhat reduced (Tab. III). With minimum coancestry mating, relationships within a population become more equal, because the least related animals are mated and with the restriction on the number of progeny per mating pair to zero or one, as in the factorial mating scheme of Woolliams [17], extreme relationships are avoided. The variance of Mendelian sampling terms increases, due to lower levels of inbreeding of the parents. These three effects give the selection algorithm an advantage at the next round of selection, such that it can select mainly on the more variable Mendelian sampling terms, i.e. there is less need to account for relationships between animals since relationships are more equal.

The effect of MC1 on $\Delta G$ for schemes with progeny testing was much smaller than for schemes without progeny testing, probably because the choices between mating pairs are reduced, due to the smaller number of selected sires. Progeny testing changed, however, the whole breeding scheme, resulting in more intense selection of older sires and a substantially increased $\Delta G$ at the same $\Delta F(35 \%$, Tabs. II and IV). Here, each sire was tested on 100 daughters. If the number of progeny tested daughters decreases, a new balance between 
the use of proven versus young sires has to be found, which might result in the selection of more young sires, and fewer proven sires.

The $\Delta G$ increased from 11 to $18 \%$ for MC1 compared with RAND mating for the scheme with overlapping generations, whereas $\Delta G$ increased from 1 to $4 \%$ for the scheme with discrete generations at the same $\Delta F$ and $\Delta G$ per generation (Tabs. II, III and V). The resulting scheme for discrete generations was, however, rather large in size, such that the effect of non-random mating was expected to be rather small $[3,15]$. Hence, MC1 mating seems more effective for schemes with overlapping generations than for schemes with discrete generations, when compared at the same rate of inbreeding per generation and similar size (number of candidates). Caballero et al. [3] found that $\Delta F$ was reduced about $50 \%$ more for schemes with overlapping generations than for discrete generations. They compared minimum coancestry mating without the restriction of zero or one progeny per mating pair and compensatory mating on the average coancestry of the candidates to all other selected animals (CMM) with random mating. CMM was more effective in reducing $\Delta F$ for less intense schemes (e.g. phenotypic selection or large populations) and minimum coancestry mating was more effective for more intense schemes (e.g. BLUP selection and small populations). We could confirm this conclusion in the study of non-random mating for discrete generations [15]. The comparison of [3] between discrete and overlapping schemes is, however, difficult to compare with our schemes. Firstly, in their study, they observed the effect of nonrandom mating in reduced $\Delta F$, whereas $\Delta F$ is restricted in our study, such that the effect of non-random mating can be seen in increased genetic response. Secondly, they did not correct for the increased size of the overlapping scheme compared with the discrete scheme. Thirdly, their discrete schemes were smaller than our schemes and had fixed contributions of the two age-classes instead of optimum contributions of ten age-classes here. Fourthly, they used BLUP selection, for which a larger effect of non-random mating is expected than for optimum contribution selection, because with BLUP selection, progeny from successful families have a higher probability of being selected due to their superior $E B V \mathrm{~s}$. A mating strategy, which mates animals from successful families (i.e. large) to animals from unsuccessful families, avoids this cumulative effect of BLUP selection on the contributions of some families. Optimum contribution selection directly controls the contributions of families. Hence, the above advantage of non-random mating does not hold for optimum contribution selection.

For this study, we compared different schemes with ten reproductive ageclasses for males and females, and females obtained only three repeated records (in order to reduce computation of $E B V \mathrm{~s}$ ). The results of this study show that the older females were hardly selected, because their $E B V \mathrm{~s}$ were much lower than the $E B V \mathrm{~s}$ of younger females. With a more stringent restriction on inbreeding, 
the older females with high $E B V \mathrm{~s}$ would probably be selected. Thus, the number of reproductive age-classes is especially important for small schemes with low inbreeding (stringent restriction), where the older animals are also competitive candidates, i.e. a more stringent $\Delta F$ restriction would reduce $\Delta G$ more if the older age-classes are not available.

\subsection{Iteration on generation intervals}

Schemes without iteration on generation intervals used much less computer time. For one replicate of 20 years with a constraint on $\Delta F$ of $0.25 \%$, RAND needed 54 min and RAND_NIT needed $11 \mathrm{~min}$ on an Alpha 500 workstation.

After the breeding schemes had stabilised, there was very little difference between the schemes with and without iteration on the distribution of parents over age-classes. However, during the first years of the scheme, when relationships in the population were not yet equalised, the level of inbreeding, and therefore the genetic level also fluctuated more for the scheme without iteration on the generation interval than for the scheme with an iteration on generation interval (Figs. 1 and 2). This makes the scheme without iteration on the generation interval useful for steady-state breeding schemes, where the generation interval is stable and approximately known. If a new breeding scheme is implemented, iteration on the generation interval is desirable such that early fluctuations on inbreeding and genetic levels are reduced.

\subsection{Practical schemes}

Optimum contribution is a dynamic selection tool, i.e. it adapts to the current situation and makes the contribution of families more equal. This makes optimum contribution selection more efficient on maximizing genetic gain with a restriction on inbreeding than a static selection tool [7,12]. For practical schemes, the actual contribution of each animal can only be aimed at. External factors may reduce or increase the actual number of progeny of a certain selected animal. However, in these cases, the next round of optimum contribution selection will adapt to the new situation in an optimal way, e.g. by increasing or decreasing the number of selected progeny in a family. Hence, there is correction of contributions over generations. Furthermore, additional (practical) restrictions can be added to the optimum contribution selection algorithm, e.g. restrictions on the maximum contribution (number of offspring) an animal can get (see Appendix of [11]).

Two factors make optimum contribution selection best suitable for $e$.g. nuclei with elite breeding animals or smaller selection lines. Firstly, the optimum contribution algorithm requires recording of pedigree and control over selection. With non-random mating schemes, the matings also have to be controlled. Secondly, optimum contribution selection performs best for small populations 
$([7,12]$ and this study). Increasing size and less stringent restrictions on inbreeding lead to schemes that resemble BLUP selection schemes, although the number of selected animals (especially sires) and generation intervals are not optimized for regular BLUP schemes as they are for optimum contribution selection.

Recommended $\Delta F$ restrictions are in the range of 0.5 through $1.3 \%$ per generation (see for example $[4,8,13]$ ), although it is difficult to generalize a figure for all situations (the $\Delta F$ restriction will also depend on the historical $\Delta F$ in the population, the attitude of the breeding company towards risk, the openness of the breeding scheme to animals from outside the scheme etc.). An alternative dynamic selection method by Wray and Goddard [18] maximizes genetic gain with a cost factor, $k$, on the average relationship $(E B V-k \mathrm{~A})$. The cost factors on inbreeding are difficult to estimate (they include $e$.g. costs due to increased risk, inbreeding depression on (non)breeding goal traits, assessing time horizons), and any failure to account for all costs results in schemes with a too high $\Delta F$. The cost factors are also more difficult to interpret in breeding practices than restrictions on $\Delta F$.

Recently, Wu and Schaeffer [19] presented a study, where the methods of Grundy et al. [5] and Verrier et al. [16], which reduce inbreeding, were compared with their new method. All three methods selected for modified $E B V \mathrm{~s}$ with an artificially reduced the effect of parent average on the animal model $E B V$ s. The advantage of these methods is that they require very few changes to current selection methods, although computer simulations are needed to find the optimum weight of the parent average on the modified $E B V \mathrm{~s}$. There are, however, four drawbacks of selection for modified $E B V \mathrm{~s}$ : 1 . The number of selected males (and females) is not optimized by these methods, whereas this is an important tool to reduce inbreeding; 2 . They do not consider the selected animals as a group, while the effect of an animal on future inbreeding depends on who else was selected; 3 . These methods rely on truncation selection, i.e. animals above a certain level threshold obtain equal contributions, which may be more practical in some cases, but it is certainly not optimal $[2,6]$; 4 . If animals have no performance records and no progeny, their $E B V$ s consist only of their parent averages and reducing the weight of the parent average would not affect the ranking of the animals.

\subsection{Conclusions}

In conclusion, MC1 mating increased genetic response from 11 to $18 \%$ compared with RAND mating for optimum contribution selection and overlapping generations. The superiority of $\mathrm{MC} 1$ increased with a more stringent constraint on $\Delta F$ and with smaller sizes of the schemes. When progeny tested bulls were available, they were selected by the optimum contribution selection algorithm, which increased genetic response. The effect of MC1 mating was, however, 
reduced. Omitting the iteration on the distribution of parents over age-classes hardly affected the resulting breeding scheme when the scheme had reached its steady-state, but fluctuated more before their steady-state was reached, such that iteration on the generation interval seems, in general, more safe. MC1 mating increases genetic response more for schemes with overlapping generations than for schemes with discrete generations.

\section{ACKNOWLEDGEMENTS}

We are grateful to Piter Bijma and Pim Brascamp for helpful comments and suggestions.

\section{REFERENCES}

[1] de Boer I.J.M., Meuwissen T.H.E., van Arendonk J.A.M., Combining genetic and clonal response in a closed dairy cattle nucleus scheme, Anim. Prod. 59 (1994) 345-358.

[2] Bondesson L., Mathematical principles of optimal utilisation of clones under diversity constraints. Report No. 27, Swedish University of Agricultural Sciences, Section of Forest Biometry, Umea, 1989.

[3] Caballero A., Santiago E., Toro M.A., Systems of mating to reduce inbreeding in selected populations, Anim. Sci. 62 (1996) 431-442.

[4] FAO, Secondary Guidelines for development of national farm animal genetic resources management plans: Management of small populations at risk. Rome, 1998.

[5] Grundy B., Caballero A., Santiago E., Hill W.G., A note on using biased parameter values and non-random mating to reduce rates of inbreeding in selection programmes, Anim. Prod. 59 (1994) 465-468.

[6] Grundy B., Villanueva B., Woolliams J.A., Dynamic selection procedures for constrained inbreeding and their consequences for pedigree development, Genet. Res. 72 (1998) 159-168.

[7] Grundy B., Villanueva B., Woolliams J.A., Dynamic selection for maximizing response with constrained inbreeding in schemes with overlapping generations, Anim. Sci. 70 (2000) 373-382.

[8] Goddard M.E., Optimal effective population size for the global population size of black and white dairy cattle, J. Dairy Sci. 75 (1992) 2902-2911.

[9] Henderson C.R., Applications of Linear Models in Animal Breeding, University of Guelph Press, Guelph, 1984.

[10] Hill W.G., Prediction and evaluation of response to selection with overlapping generations, Anim. Prod. 18 (1974) 117-139.

[11] Meuwissen T.H.E., Maximizing the response of selection with a predefined rate of inbreeding, J. Anim. Sci. 75 (1997) 934-940.

[12] Meuwissen T.H.E., Sonesson A.K., Maximizing the response of selection with a predefined rate of inbreeding: Overlapping generations, J. Anim. Sci. 76 (1998) 2575-2583. 
[13] Meuwissen T.H.E, Woolliams J.A., Effective sizes of livestock populations to prevent a decline in fitness, Theor. Appl. Genet. 89 (1994) 1019-1026.

[14] Press W.H., Flannery B.P., Teukolsky S.A., Vetterling W.T., Minimization or maximization of functions, in: Numerical recipes - The art of scientific computing. University of Cambridge Press, 1989, pp. 274-334.

[15] Sonesson A.K., Meuwissen T.H.E., Mating schemes for optimum contribution selection with constrained rates of inbreeding, Genet. Sel. Evol. 32 (2000) 231248.

[16] Verrier E., Colleau J.J., Foulley J.L., Long-term effects of selection based on the animal model BLUP in a finite population, Theor. Appl. Genet. 87 (1993) 446-454.

[17] Woolliams J.A., Modifications to MOET nucleus breeding schemes to improve rates of genetic progress and decrease rates of inbreeding in dairy cattle, Anim. Prod. 49 (1989) 1-14.

[18] Wray N.R., Goddard M.E., Increasing long term response to selection, Genet. Sel. Evol. 26 (1994) 431-451.

[19] Wu L., Schaeffer L.R., Reducing the effect of parent averages from animal solutions in mixed model equations, J. Anim. Breed. Genet. 117 (2000) 361-374.

To access this journal online: www.edpsciences.org 